\title{
Camptocormia: As the First Sign of Parkinson's Disease
}

\author{
Yoon-Sang Oh, Joong-Seok Kim, Sung-Woo Chung, Young-Do Kim, Kwang-Soo Lee
}

Can J Neurol Sci. 2011; 38: 370-372

Camptocormia is defined as an abnormal flexion of the thoracolumbar spine and trunk that appears while standing or walking ${ }^{1}$. A severe trunk flexion was noted in the original description of Parkinson disease (PD), and more recently has been described in other basal ganglia diseases, dystonia, and neuromuscular disorders. A recent epidemiological study revealed that this is a relatively common symptom and seems to be related to the clinical severity of $\mathrm{PD}^{2}$.

Here, we report a patient who had a marked camptocormia prior to the onset of clinical triad of Parkinsonism.

\section{CASe}

A 77-year-old woman was admitted to our hospital with complaints of a right side tremor at rest, bradykinesia and gait disturbance. The patient had been well until about four months earlier, when she began to notice a bend in her spine that was present while standing or walking. This abnormal contraction was relieved on sitting and supine position. Soon thereafter, she felt her gait was mildly uncomfortable. At that time, the patient visited a private medical clinic and was diagnosed as kyphosis due to aging process. Although a formal neurological examination was not recorded in the medical record, there was no mentioned about tremor, bradykinesia, or rigidity to suggest Parkinsonism. Two months before admission, the patient developed a masked face, dysarthria, bradykinesia, and right hand rest tremors. Over a period of weeks, the patient began to stand without assistance and could take a few steps, bent forward with short step and stride. The patient reported no history of spine trauma, surgical incidents, or neurological diseases. There was no exposure to neuroleptic medications.

On examination, the patient was oriented to place and people, but had difficulty copying, calculating, and maintaining attention. A neuropsychological investigation using Seoul Neuropsychological Screening Battery ${ }^{3}$ showed a mild memory impairment, reduced psychomotor speed, and visuospatial dysfunction. In addition, the patient exhibited hypomimia and mild dysarthria. All four limbs exhibited bradykinesia and rigidity. The patient stood on a narrow base, and her stride was reduced with decreased arm swing bilaterally. The posture was markedly stooped: nearly $60^{\circ}$ with painless forward trunk flexion while standing and walking, but the flexed posture was alleviated in the sitting position (see the supplementary video online). On the modified Hoehn \& Yahr staging test, the patient scored 2.5 for the non-medicated state and the result of the Unified Parkinson's Disease Rating Scale (UPDRS) total was 37.

The following laboratory test results were negative or normal: biochemical and hematologic profiles, thyroid function testing, and EMG on the thoracolumbar paraspinal muscles. Diffuse osteopenia was found on the plain lumbar spine series. Magnetic resonance imaging of the brain showed mild multifocal subcortical deep white matter hyperintensity and the positron emission tomography using ${ }^{18} \mathrm{~F}-\mathrm{N}$-(3-fluoropropyl)-2betacarbon ethoxy-3beta-(4-iodophenyl) nortropane (FP-CIT) showed bilateral decreased striatal dopamine binding uptake compared with age-matched healthy control subject (Figure 1). Myocardial ${ }^{123}$ I-metaiodibenzylguanidine (MIBG) scintigraphy, six months after treatment, showed severe postganglionic sympathetic denervation (Figure 2)

The patient was treated initially with levodopa/carbidopa/ entacarpone 50/12.5/200 mg twice daily and pramipexole 0.25 mg twice daily, with marked improvement of the bradykinesia and rigidity. The levodopa dose was slowly increased to $150 \mathrm{mg}$ three times a day over three months, and the patient noted significant improvement of the camptocormia (see the supplementary video online at www.cjns.org). The Hoehn \& Yahr and UPDRS total, was 1 and 14 for the on-period.

\section{Discussion}

Initially, most patients with PD complain of the classic motor triad of symptoms: a resting tremor, bradykinesia and cogwheel rigidity. In addition, a mild stooped posture is a hallmark of Parkinsonism; extreme trunk forward flexion is not common. In very few cases, camptocormia occurs early; more frequently it is a manifestation of advanced disease ${ }^{4}$. A recent clinical study suggested that camptocormia was more common in patients with more severe PD, on longer levodopa treatment duration, with greater levodopa daily doses, and with dementia ${ }^{2}$. However, some have described conflicting results ${ }^{5}$.

The pathophysiology of this condition is not clear, although two provisional mechanisms have been proposed; a central disorder or an outcome of peripheral mechanism ${ }^{6}$. Since some patients respond to dopaminergic therapy, it may be due to a dopamine deficiency observed with extrapyramidal

From the Department of Neurology, The Catholic University of Korea, Seoul St. Mary's Hospital, Seoul, Korea.

Received June 11, 2010. Final Revisions Submitted September 23, 2010 Correspondence to: Joong-Seok Kim, Department of Neurology, Seoul St. Mary's Hospital, 505, Banpo-dong, Seocho-gu, Seoul, 137-701, South Korea. 


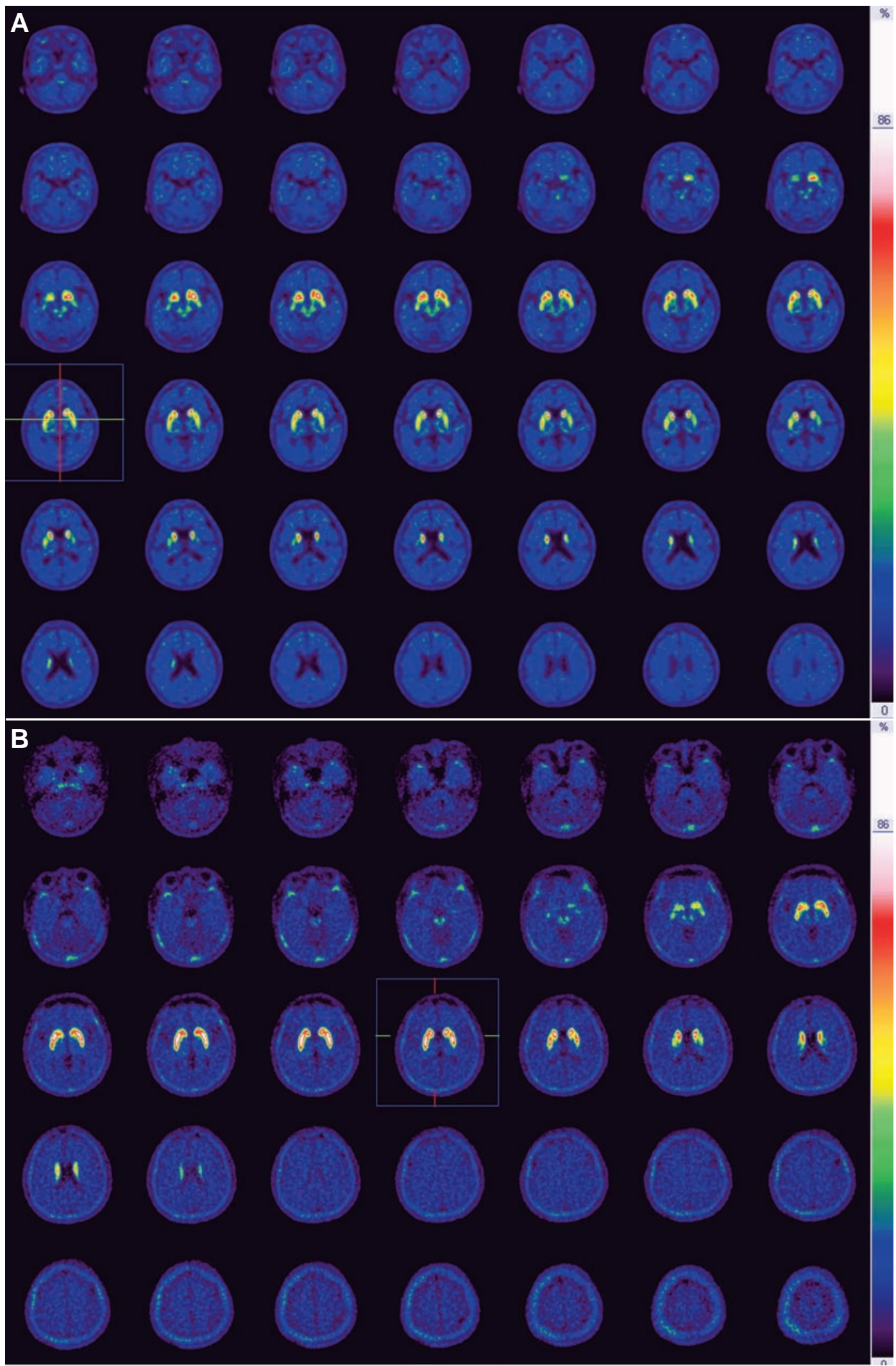

Figure 1: The positron emission tomography using ${ }^{18} \mathrm{~F}$ - $\mathrm{N}$-(3-fluoropropyl)-2beta-carbon ethoxy-3beta-(4-iodophenyl) nortropane (FP-CIT) showed bilateral decreased striatal dopamine binding uptake more marked the putamen (A) compared with healthy control subject $(B)$. 

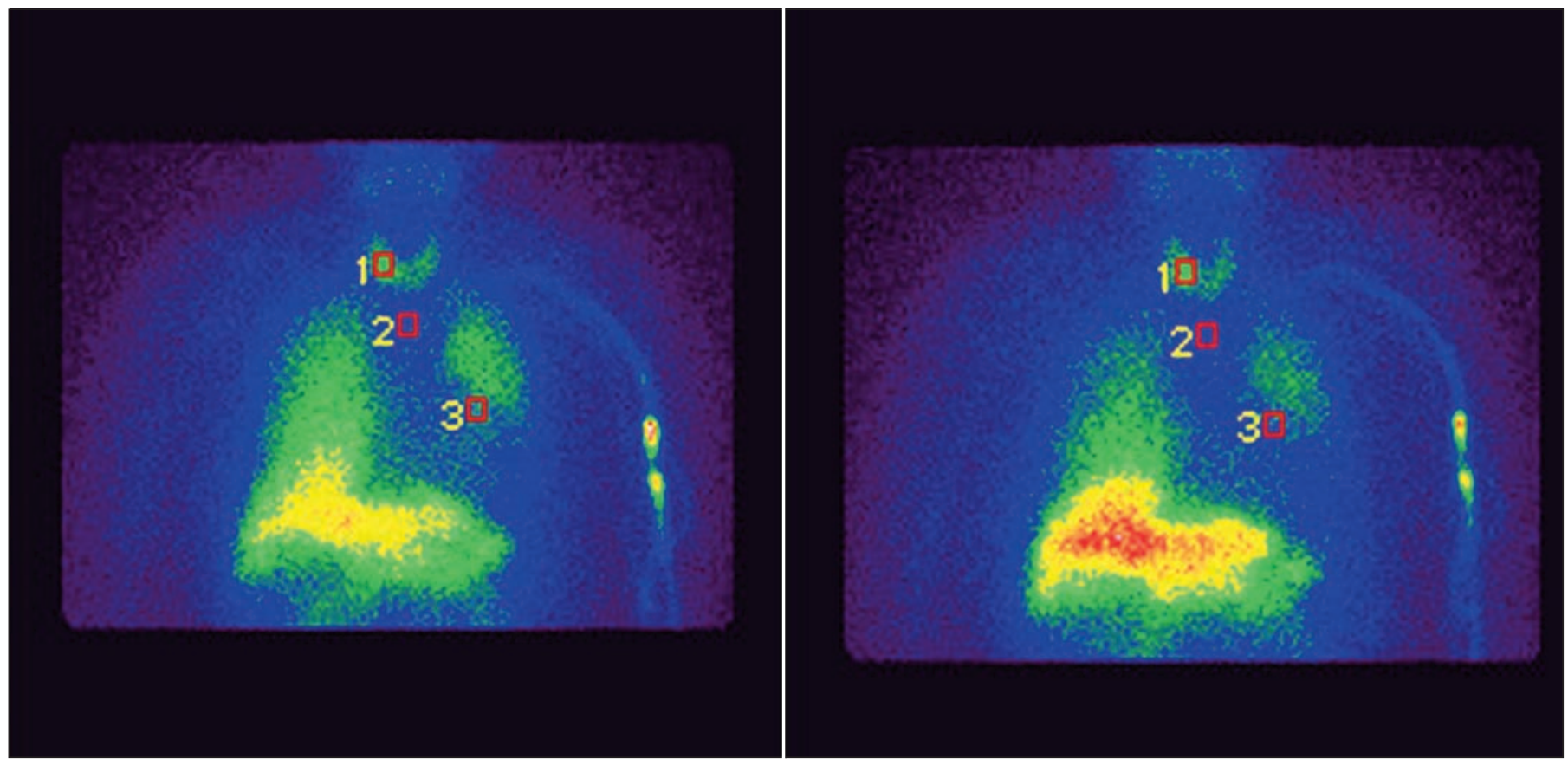

Figure 2: Myocardial ${ }^{123}$ I-metaiodibenzylguanidine $(M I B G)$ scintigraphy of the patient showed severe postganglionic sympathetic denervations in the early $(A)$ and delayed images $(B)$. The heart to mediastinum ratio is 1.35 in the early image and 1.23 in the delayed image.

symptoms ${ }^{1,7,8}$. However, the benefits of levodopa are very limited; many patients do not have obvious benefits, and levodopa worsened the condition in some patients suggesting additional, non-dopaminergic neuronal dysfunction in the basal ganglia $^{4,9}$.

It should be ruled out the probability of atypical parkinsonisms with axial dystonia, because the clinical course in this patient was very rapid. However, the clear evidence of functional neuroimaging and the excellent response to levodopa were suggestive of idiopathic PD. Therefore, we think this case is the first to show camptocormia as the initial sign of PD.

\section{ACKNOWLEDGEMENT}

The authors thank Lee, Ho Jae for help in English editing.

\section{REFERENCES}

1. Djaldetti R, Mosberg-Galili R, Sroka H, Merims D, Melamed E. Camptocormia (bent spine) in patients with Parkinson's disease characterization and possible pathogenesis of an unusual phenomenon. Mov Disord. 1999;14(3):443-7.

2. Tiple D, Fabbrini G, Colosimo C, et al. Camptocormia in Parkinson disease: an epidemiological and clinical study. J Neurol Neurosurg Psychiatry. 2009;80(2):145-8.

3. Kang Y, Na DL. Seoul Neuropsychological Screening Battery (SNSB) Seoul; Human Brain Research \& Consulting Co. 2003.

4. Melamed E, Djaldetti R. Camptocormia in Parkinson's disease. J Neurol. 2006;253(S7):VII14-6.

5. Lepoutre AC, Devos D, Blanchard-Dauphin A, et al. A specific clinical pattern of camptocormia in Parkinson's disease. J Neurol Neurosurg Psychiatry. 2006;77(11):1229-34.

6. Jankovic J. Camptocormia, head drop and other bent spine syndromes: heterogeneous etiology and pathogenesis of Parkinsonian deformities. Mov Disord. 2010;25(5):527-8.

7. Van Gerpen JA. Dopa-responsive dystonic camptocormia. Neurology. 2006;66(11):1779.

8. Song IU, Kim JS, Lee KS. Dopa-responsive camptocormia in a patient with multiple system atrophy. Parkinsonism Relat Disord. 2008;14(2): 161-3.

9. Bloch F, Houeto JL, Tezenas du Montcel S, et al. Parkinson's disease with camptocormia. J Neurol Neurosurg Psychiatry. 2006;77(11):1223-8. 